\title{
CD8 coreceptor-mediated focusing can reorder the agonist hierarchy of peptide ligands recognized via the $\mathrm{T}$ cell receptor
}

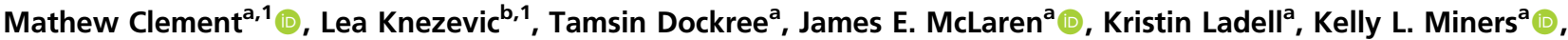

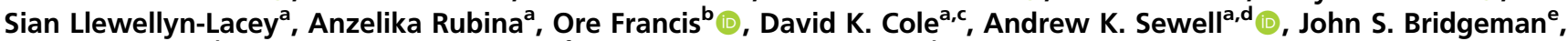 \\ David A. Price ${ }^{a, d}{ }_{(D)}$ Hugo A. van den Berg ${ }^{\text {f } 1}$ (D), and Linda Wooldridge ${ }^{b, 1,2}(\mathbb{D}$

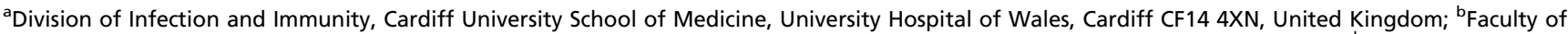
Health Sciences, University of Bristol, Bristol BS8 1TD, United Kingdom; ' Immunocore Ltd., Abingdon OX14 4RY, United Kingdom; ${ }^{\mathrm{d} S y s t e m s}$ Immunity Research Institute, Cardiff University School of Medicine, University Hospital of Wales, Cardiff CF14 4XN, United Kingdom; ' Immetacyte Ltd., Manchester M13 9XX, United Kingdom; and 'Warwick Mathematics Institute, University of Warwick, Coventry CV4 7AL, United Kingdom
\end{abstract}

Edited by Peter Parham, Stanford University School of Medicine, Stanford, CA, and accepted by Editorial Board Member Peter Cresswell June 8, 2021 (received for review September 17, 2020)

$\mathrm{CD}^{+} \mathrm{T}$ cells are inherently cross-reactive and recognize numerous peptide antigens in the context of a given major histocompatibility complex class I $(\mathrm{MHCl})$ molecule via the clonotypically expressed T cell receptor (TCR). The lineally expressed coreceptor CD8 interacts coordinately with $\mathrm{MHCl}$ at a distinct and largely invariant site to slow the TCR/peptide-MHCl (pMHCl) dissociation rate and enhance antigen sensitivity. However, this biological effect is not necessarily uniform, and theoretical models suggest that antigen sensitivity can be modulated in a differential manner by CD8. We used two intrinsically controlled systems to determine how the relationship between the TCR/pMHCl interaction and the $\mathrm{pMHCl} / \mathrm{CD} 8$ interaction affects the functional sensitivity of antigen recognition. Our data show that modulation of the $\mathrm{pMHCl} / \mathrm{CD} 8$ interaction can reorder the agonist hierarchy of peptide ligands across a spectrum of affinities for the TCR.

CD8 coreceptor | pMHCI | T cell activation

C $8^{+} \mathrm{T}$ cells are critical for protective immunity against intracellular pathogens and various tumors. At the molecular level, activation is triggered by foreign or mutated peptide fragments presented on the cell surface by major histocompatibility complex class I (MHCI) molecules, which act as ligands for the somatically rearranged $\mathrm{T}$ cell receptor (TCR) and the germline-encoded coreceptor CD8 $(1,2)$. The clonotypically expressed TCR confers antigen specificity by interacting with the peptide-binding platform of MHCI, which comprises the $\alpha 1$ and $\alpha 2$ domains, whereas the lineally expressed coreceptor CD8 is known to enhance antigen sensitivity by interacting primarily with the $\alpha 3$ domain of MHCI (3-7). This latter interaction is biophysically and spatially independent of peptide-MHCI (pMHCI) engagement via the TCR (8). However, the largely invariant nature of the $\mathrm{pMHCI} / \mathrm{CD} 8$ interaction does not necessarily translate into a uniform gain of function, and theoretical studies have suggested that antigen sensitivity can be modulated in a differential manner, potentially altering the agonist hierarchy of peptide ligands for any given TCR (9-11).

The pMHCI/CD8 interaction slows the dissociation rate of the TCR/pMHCI interaction $(9,12)$. Functional sensitivity depends nonmonotonically on this dissociation rate (13), as long as the system is limited by MHCI $(10,14,15)$. The nature of this relationship implies that functional sensitivity reaches a maximum at a particular dissociation rate. Strong agonists are relatively insensitive to modulation of the dissociation rate, because the curve has a negligible slope in the vicinity of the optimal value. In contrast, weak agonists are typically characterized by faster dissociation rates, modulation of which markedly alters functional sensitivity (16). Accordingly, the pMHCI/CD8 interaction generally acts to increase agonist potency, maximizing the number of peptide ligands that can be recognized via a given TCR. However, theoretical models predict that ligands with dissociation rates below or close to the optimal value will respond differently, amounting to a differential focusing effect, whereby strong agonists can become less potent at dissociation rates beyond the optimal value. If operative in vivo, such an effect could allow individual clonotypes to focus on salient ligands (9), reconciling the inherent need for cross-reactivity with the inherent need for specificity (17).

We used two monoclonal systems incorporating biophysically defined peptide ligands and variants of MHCI with altered coreceptorbinding properties to test the differential focusing hypothesis experimentally. In line with earlier predictions, we found that modulation of the $\mathrm{pMHCI} / \mathrm{CD} 8$ interaction reordered the agonist hierarchy of peptide ligands recognized via the TCR.

\section{Results}

To test the notion that coreceptor-mediated effects can alter ligand specificity, we determined the extent to which CD8 modulated functional responses initiated via the MEL5 TCR, which recognizes the heteroclitic Melan-A epitope ELAGIGILTV 26 -35/A27L (ELA) restricted by HLA-A*0201 (abbreviated from hereon as HLA-A2). Ligand recognition in this system has been characterized previously using surface plasmon resonance $(18,19)$. Biophysically defined peptide ligands, including a weak agonist (3T), the wild-type epitope (ELA), and a superagonist (FAT), were selected for

\section{Significance}

Sufficient immune coverage of the peptide universe within a finite host requires highly degenerate T cell receptors (TCRs). However, this inherent need for antigen cross-recognition is associated with a high risk of autoimmunity, which can only be mitigated by a process of adaptable specificity. We describe a mechanism that resolves this conundrum by allowing individual clonotypes to focus on specific peptide ligands without alterations to the structure of the TCR.

Author contributions: M.C., L.K., T.D., J.E.M., K.L.M., S.L.-L., A.R., O.F., and D.K.C. performed research; A.K.S. and J.S.B. provided key reagents; K.L., D.A.P., H.A.v.d.B., and L.W. supervised research; and D.A.P., H.A.v.d.B., and L.W. wrote the paper.

The authors declare no competing interest.

This article is a PNAS Direct Submission. P.P. is a guest editor invited by the Editorial Board.

This open access article is distributed under Creative Commons Attribution License 4.0 (CC BY)

${ }^{1}$ M.C., L.K., H.A.v.d.B., and L.W. contributed equally to this work

${ }^{2}$ To whom correspondence may be addressed. Email: linda.wooldridge@bristol.ac.uk.

This article contains supporting information online at https://www.pnas.org/lookup/suppl/ doi:10.1073/pnas.2019639118/-/DCSupplemental.

Published July 16, 2021 
the purposes of this work to introduce a range of TCR/pMHCI affinities (Table 1). C1R cells expressing HLA-A2 D227K/T228A, which abrogates the coreceptor interaction (20), wild-type HLAA2, HLA-A2 A245V/K $\mathrm{K}^{\mathrm{b}}$, which enhances the coreceptor interaction (21), or HLA-A2 $\mathrm{K}^{\mathrm{b}}$, which superenhances the coreceptor interaction (22), were used in parallel to introduce a range of pMHCI/CD8 affinities (Table 2). Importantly, surface plasmon resonance experiments have shown that none of these mutations, namely D227K/T228A, A245V/K $\mathrm{K}^{\mathrm{b}}$, and $\mathrm{K}^{\mathrm{b}}$, affect the TCR/pMHCI interaction $(12,21)$.

In preliminary experiments, we quantified CD69 on the surface of MEL5 TCR ${ }^{+} \mathrm{CD}^{+}$J.RT3-T3.5 cells as a measure of activation in response to 3T, ELA, or FAT presented in the context of HLAA 2 D227K/T228A, wild-type HLA-A2, or HLA-A2 A245V/K ${ }^{\mathrm{b}}$ (SI Appendix, Figs. S1 and S2). Functional sensitivity was determined as the $p \mathrm{EC}_{50}$ value for each parameter combination (Fig. $1 A$ ). In the absence of a pMHCI/CD8 interaction (HLA-A2 D227K/T228A), activation was a simple function of TCR/pMHCI affinity (Fig. $1 A$ and SI Appendix, Figs. S1 and S2). The agonist potencies of 3T and ELA were enhanced in the context of HLA-A2 and HLA-A2 $\mathrm{A} 245 \mathrm{~V} / \mathrm{K}^{\mathrm{b}}$ relative to HLA-A2 D227K/T228A (Fig. $1 A$ ). In contrast, the agonist potency of FAT was only marginally enhanced in the context of HLA-A2 relative to HLA-A2 D227K/T228A and, consistent with the notion of an optimal activation window, decreased slightly in the context of HLA-A2 A245V/K $\mathrm{K}^{\mathrm{b}}$ relative to HLA-A2 (Fig. $1 A$ and SI Appendix, Fig. S3A). As a consequence, the agonist potency of FAT relative to the agonist potency of ELA was reduced at higher pMHCI/CD8 affinities (Fig. $1 B$ ), and in three of four replicate experiments, ELA was the most potent ligand in the context of HLA-A2 and HLA-A2 A245V/K ${ }^{\mathrm{b}}$ (Fig. $1 B-E$ ).

To confirm these findings, we quantified the production of IFN- $\gamma$ by clonal MEL5 CD8 ${ }^{+} \mathrm{T}$ cells in response to 3T, ELA, or FAT presented in the context of HLA-A2 D227K/T228A, wildtype HLA-A2, or HLA-A2 $\mathrm{K}^{\mathrm{b}}$ (SI Appendix, Figs. S4 and S5). Functional sensitivity was again determined as the $p \mathrm{EC}_{50}$ value for each parameter combination (Fig. $2 A$ ). The activation data were largely analogous to those obtained with MEL5 $\mathrm{TCR}^{+}$ $\mathrm{CD}^{+}$J.RT3-T3.5 cells. In particular, the agonist potency of FAT was enhanced in the context of HLA-A2 relative to HLAA2 D227K/T228A and decreased slightly in the context of HLAA $2 \mathrm{~K}^{\mathrm{b}}$ relative to HLA-A2 (Fig. $2 A$ and SI Appendix, Fig. S3B), mirroring the downturn in functional sensitivity observed with MEL5 TCR ${ }^{+} \mathrm{CD}^{+}{ }^{+}$J.RT3-T3.5 cells in the context of HLA-A2 $\mathrm{A} 245 \mathrm{~V} / \mathrm{K}^{\mathrm{b}}$ relative to HLA-A2 (Fig. $1 A$ and SI Appendix, Fig. $\mathrm{S} 3 A$ ). As a consequence, the agonist potency of FAT relative to the agonist potency of ELA was again reduced at higher pMHCI/ CD8 affinities (Fig. 2B), and in three of four replicate experiments, ELA was the most potent ligand in the context of HLAA $2 \mathrm{~K}^{\mathrm{b}}$ (Fig. $2 B-E$ ).

In further experiments, we quantified the production of IFN- $\gamma$ and MIP-1 $\beta$ by clonal MEL5 CD8 ${ }^{+} \mathrm{T}$ cells in response to ELA or 1I, a superagonist characterized for the purposes of this work, presented in the context of HLA-A2 D227K/T228A, wild-type HLA-A2, or HLA-A2 $\mathrm{K}^{\mathrm{b}}$ (Table 1 and SI Appendix, Fig. S6). Irrespective of the functional readout, 1I was the more potent agonist in the context of HLA-A2 D227K/T228A and HLA-A2,

Table 1. TCR/pMHCl dissociation constants and kinetics for agonists of the MEL5 TCR

\begin{tabular}{lcccc} 
MHCl & Epitope & $\mathrm{k}_{\text {on }}\left(\mathrm{M}^{-1} \mathrm{~s}^{-1}\right)$ & $\mathrm{k}_{\text {off }}\left(\mathrm{s}^{-1}\right)$ & $\mathrm{K}_{\mathrm{D}}(\mu \mathrm{M})$ \\
\hline HLA-A2 & ELTGIGILTV (3T) & $\sim 1.2 \times 10^{4}$ & $\sim 1$ & $82 \pm 4$ \\
HLA-A2 & ELAGIGILTV (ELA) & $\sim 4.4 \times 10^{4}$ & $\sim 0.75$ & $17 \pm 1$ \\
HLA-A2 & FATGIGIITV (FAT) & $\sim 1.2 \times 10^{5}$ & 0.36 & $3 \pm 1$ \\
HLA-A2 & ILAGIGILTV (1I) & $\sim 1.3 \times 10^{5}$ & $\sim 1.3$ & $10.2 \pm 0.7^{*}$
\end{tabular}

*Surface plasmon resonance data are shown in SI Appendix, Fig. S6A.
Table 2. $\mathrm{pMHCl} / \mathrm{CD} 8$ dissociation constants for variants of HLA-A2

\begin{tabular}{lcc} 
MHCl & Mutation & $\mathrm{K}_{\mathrm{D}}(\mu \mathrm{M})$ \\
\hline HLA-A2 D227K/T228A & $\mathrm{MHCl} \alpha 3$ domain & $>10,000$ \\
HLA-A2 WT & $\mathrm{N} / \mathrm{A}$ & $137 \pm 9.7$ \\
HLA-A2 A245V/K & $\mathrm{MHCl} \alpha 3$ domain & $27 \pm 1$ \\
HLA-A2 $\mathrm{K}^{\mathrm{b}}$ & $\mathrm{MHCl} \alpha 3$ domain & 11
\end{tabular}

N/A, not applicable.

whereas ELA was the more potent agonist in the context of HLA-A2 $\mathrm{K}^{\mathrm{b}}$, thereby eliminating the possibility of a spurious link between the observed differential focusing effect and the anchor residue mutation present in FAT (SI Appendix, Fig. S6).

To confirm these findings in another intrinsically controlled system, we used a similar approach to determine the extent to which CD8 modulated functional responses initiated via the ILA1 TCR, which recognizes the human telomerase reverse transcriptase epitope ILAKFLHWL ${ }_{540-548}$ (ILA) restricted by HLA-A2. Biophysically defined peptide ligands, including a weak agonist (5Y), the wild-type epitope (ILA), and two superagonists (3G and 3G8R), were again selected for the purposes of this work to introduce a range of TCR/pMHCI affinities (SI Appendix, Table S1). Irrespective of the functional readout, the agonist potencies of $5 \mathrm{Y}$ and ILA were enhanced in the context of HLA-A2 and HLA-A2 $\mathrm{K}^{\mathrm{b}}$ relative to HLA-A2 D227K/T228A, whereas the agonist potencies of $3 \mathrm{G}$ and $3 \mathrm{G} 8 \mathrm{R}$ were only marginally enhanced in the context of HLA-A2 relative to HLA-A2 D227K/ $\mathrm{T} 228 \mathrm{~A}$ and decreased slightly in the context of HLA-A2 $\mathrm{K}^{\mathrm{b}}$ relative to HLA-A2 (SI Appendix, Figs. S7 and S8). As a consequence, ILA was the most potent ligand in the context of HLA-A $2 \mathrm{~K}^{\mathrm{b}}$, thereby validating the general applicability of differential focusing beyond the idiosyncratic properties of a singular TCR (SI Appendix, Figs. S7 and S8).

Collectively, these results can be interpreted and understood in biological terms if two key assumptions are made: 1) functional sensitivity depends nonmonotonically on the TCR/pMHCI dissociation rate (13); and 2) the $\mathrm{pMHCI} / \mathrm{CD} 8$ interaction affects the TCR/pMHCI dissociation rate by an invariant factor, equivalent to translation on a logarithmic scale (Fig. 3). In this scenario, ligands that are recognized poorly in the absence of a $\mathrm{pMHCI} / \mathrm{CD} 8$ interaction become more potent in the presence of a physiological $\mathrm{pMHCI} / \mathrm{CD} 8$ interaction and achieve optimal agonist potency in the presence of a supraphysiological $\mathrm{pMHCI} / \mathrm{CD} 8$ interaction, whereas ligands that are recognized strongly in the absence of a pMHCI/CD8 interaction straddle an optimum in the presence of a physiological $\mathrm{pMHCI} / \mathrm{CD} 8$ interaction and become less potent in the presence of a supraphysiological pMHCI/CD8 interaction. Accordingly, the agonist hierarchy of peptide ligands, which is dictated in isolation by the TCR/pMHCI interaction, can be reordered as a function of coengagement by CD8.

\section{Discussion}

$\mathrm{CD}^{+} \mathrm{T}$ cells are inherently promiscuous and can recognize more than a million different peptide ligands via the TCR (23-26). It is well established that CD8 can enhance the functional sensitivity of antigen recognition, but in any given monoclonal system, it does not necessarily follow that CD8 will affect the agonist potency of every cognate ligand in a similar manner. Indeed, theoretical studies have suggested that the agonist hierarchy of peptide ligands can be modified or even reversed across a range of pMHCI/CD8 affinities, such that a differential focusing effect acts to optimize the recognition of particular ligands in the context of an individual TCR $(9,10,15)$. Our data provide experimental confirmation of these predictions. 


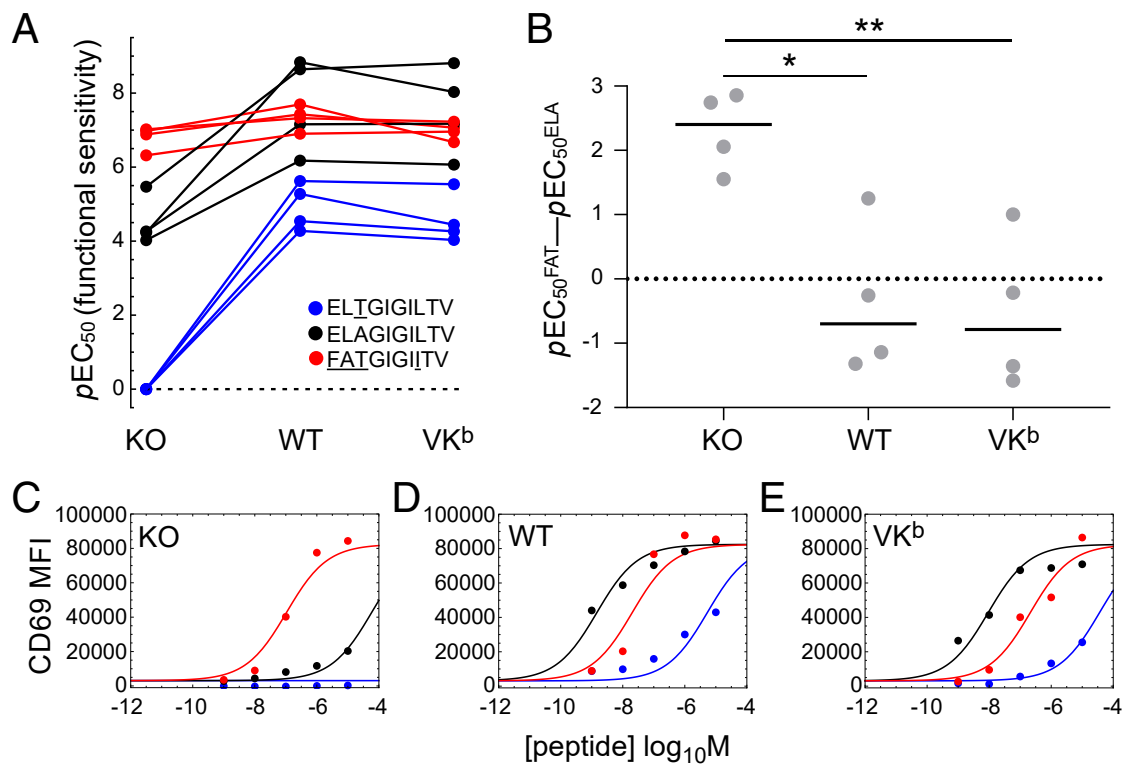

Fig. 1. CD8 reorders the agonist hierarchy of peptide ligands that induce the expression of CD69. MEL5 TCR ${ }^{+} C D 8^{+}$J.RT3-T3.5 cells were activated for $6 \mathrm{~h}$ with C1R cells expressing comparable levels of HLA-A2 D227K/T228A (KO), wild-type HLA-A2 (WT), or HLA-A2 A245V/K ${ }^{\mathrm{b}}$ (VK ${ }^{\mathrm{b}}$ ) pulsed with various concentrations of 3T (blue), ELA (black), or FAT (red). Surface expression of CD69 was measured via flow cytometry. (A) Functional sensitivity ( $p E C_{50}$ ) for each peptide ligand in the context of each $\mathrm{MHCl}$. Four replicate experiments are shown. The value for 3T in the context of HLA-A2 D227K/T228A was set to zero for graphical purposes and treated as missing data for statistical purposes. $P<0.0001$ for the ligand effect and $P<0.0001$ for the MHCl effect (two-way ANOVA with Tukey's post hoc test). (B) The agonist potency of FAT relative to the agonist potency of ELA expressed as $p E C_{50}{ }^{\mathrm{FAT}}-p \mathrm{EC}_{50}{ }^{\mathrm{ELA}}$, which is equivalent to the logarithm of the fold difference in functional sensitivity. Four replicate experiments are shown. Horizontal bars indicate median values. $* P<0.05$ and $* * P<$ 0.01 (one-way ANOVA with Tukey's post hoc test). (C-E) Representative peptide titration experiment used to calculate the parameters in $A$ and $B$. Curves were fitted in Mathematica. All four replicate experiments are shown in SI Appendix, Figs. S1 and S2.

The biological relevance of differential focusing remains unknown, but hypothetical considerations suggest that such an effect may be advantageous in vivo, especially if accompanied by feedback mechanisms that enable the process of specificity adjustment to converge on a foreign antigen. Optimal recognition of a particular agonist in this manner would maximize immune efficacy during the process of clonal expansion and simultaneously minimize the risk of autoimmunity. Affinity maturation subserves an equivalent function in B cells. In more general terms, differential focusing also provides a solution to the "Mason paradox," allowing a high degree of immune specificity alongside sufficient coverage of the peptide universe within a relatively small naive repertoire via the incorporation of degenerate TCRs (17).

Although it remains to be determined how differential focusing could operate in vivo and to what extent this might occur throughout the lifespan of any given clonotype, elegant studies have already provided important mechanistic clues. For example, double-positive thymocytes can transcriptionally down-regulate CD8 (27), and antigen encounters in the periphery can dynamically modulate clonal responsiveness via the selective internalization of CD8 (28). In addition, coreceptor use can be switched between the functionally distinct isoforms CD8- $\alpha \alpha$ and CD8- $\alpha \beta$ (29), which are further modifiable via glycosylation (30-32), and cytokine signals can transcriptionally alter the expression of CD8 (33). All of these processes affect the signaling threshold for activation via the TCR in a manner akin to affinity variation in the $\mathrm{pMHCI} / \mathrm{CD} 8$ interaction $(12,34)$. Accordingly, functional sensitivity depends on the kinetics of signalosome development $(9,10)$, which is determined by agonist potency and regulated by CD8 (35).

In line with earlier theoretical predictions, the data presented here show that agonist potency, quantified in terms of functional sensitivity, can be differentially modulated across a range of $\mathrm{TCR} / \mathrm{pMHCI}$ affinities by CD8. If this phenomenon occurs in vivo, as suggested by previous mechanistic studies, then immune reactivity could be focused on individual peptide ligands in the context of antigen-driven clonal expansions. On the basis of these collective observations, we propose that specificity adjustment operates at the level of individual clonotypes to safeguard the host in the face of an ongoing immune response, simultaneously facilitating the targeted delivery of effector functions and mitigating the risk of bystander damage, which can be triggered by inherently degenerate and therefore potentially autoreactive TCRs.

\section{Materials and Methods}

Cells. MEL5 TCR ${ }^{+} \mathrm{CD}^{+}$J.RT3-T3.5 cells were maintained in Roswell Park Memorial Institute (RPMI) 1640 medium containing $100 \mathrm{U} / \mathrm{mL}$ penicillin, $100 \mu \mathrm{g} / \mathrm{mL}$ streptomycin, $2 \mathrm{mM}$ L-glutamine, and $10 \%$ heat-inactivated fetal calf serum (all from Thermo Fisher Scientific) (R10). Clonal MEL5 and ILA1 $\mathrm{CD}^{+} \mathrm{T}$ cells were maintained in R10 supplemented with $200 \mathrm{IU} / \mathrm{mL} \mathrm{IL-2}$ and $25 \mathrm{ng} / \mathrm{mL}$ IL-15 (both from PeproTech). The MEL5 TCR is specific for the heteroclitic HLA-A2-restricted Melan-A epitope $\operatorname{ELA}(18,36)$, and the ILA1 TCR is specific for the HLA-A2-restricted human telomerase reverse transcriptase epitope ILA (37). Human embryonic kidney (HEK) 293 cells were grown in Dulbecco's modified Eagle's medium (Sigma-Aldrich) supplemented with $100 \mathrm{U} / \mathrm{mL}$ penicillin, $100 \mu \mathrm{g} / \mathrm{mL}$ streptomycin, $2 \mathrm{mM} \mathrm{L-glutamine,} \mathrm{10 \%}$ heat-inactivated fetal calf serum, and $10 \mathrm{mM}$ HEPES (all from Thermo Fisher Scientific). C1R cells expressing comparable levels of HLA-A2 or variants thereof were generated and maintained as described previously (38).

Peptides. All peptides were synthesized at $>95 \%$ purity using standard Fmoc chemistry (BioSynthesis Inc.).

Lentiviruses. The $\alpha$ and $\beta$ chains of the MEL5 TCR were engineered to contain mouse constant domains (39) and cloned into a single pSF-Lenti-EF-1 $\alpha$ lentiviral vector (Oxford Genetics) separated by a P2A sequence (Genewiz). The $\alpha$ and $\beta$ chains of CD8 were cloned similarly into a single pSF-Lenti-EF- $\alpha$ lentiviral vector (Oxford Genetics) separated by an internal ribosomal entry site sequence (Genewiz). HEK 293 cells were cotransfected with the MEL5 TCR or CD8- $\alpha \beta$ lentiviral vectors and the packaging plasmids pMDLg/pRRE, pRSV-Rev, and 

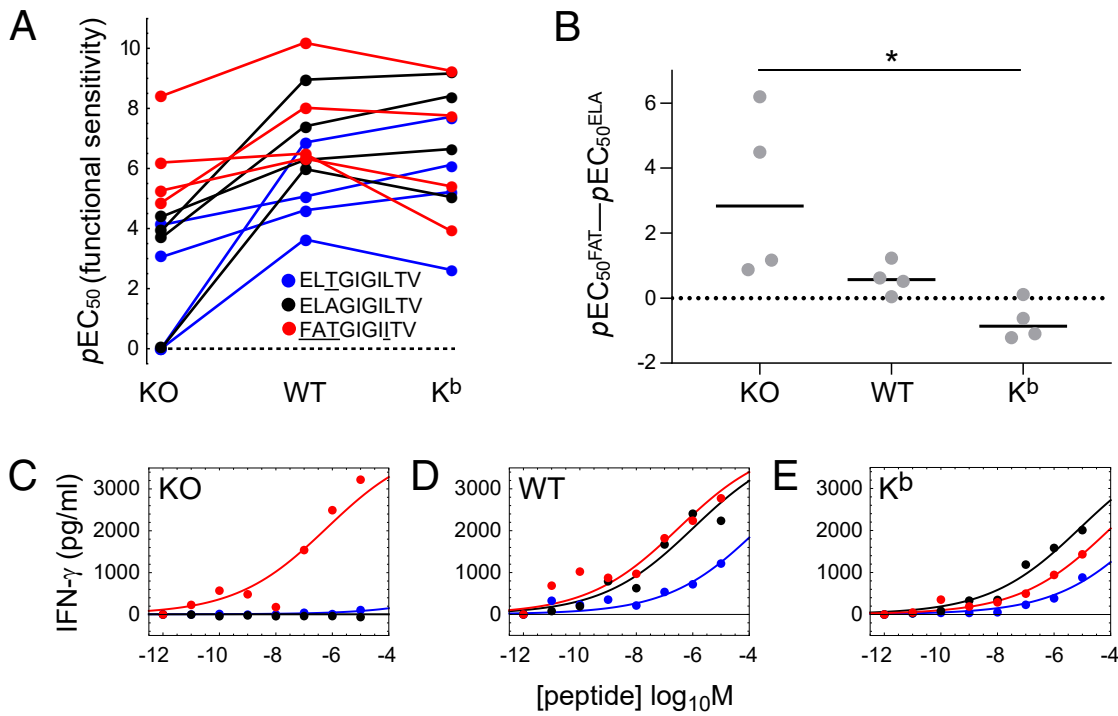

Fig. 2. CD8 reorders the agonist hierarchy of peptide ligands that induce the production of IFN- $\gamma$. Clonal MEL5 CD8 ${ }^{+} \mathrm{T}$ cells were activated for $4 \mathrm{~h}$ with $\mathrm{C} 1 \mathrm{R}$ cells expressing comparable levels of HLA-A2 D227K/T228A (KO), wild-type HLA-A2 (WT), or HLA-A2 $\mathrm{K}^{\mathrm{b}}\left(\mathrm{K}^{\mathrm{b}}\right)$ pulsed with various concentrations of $3 T$ (blue), ELA (black), or FAT (red). Secretion of IFN- $\gamma$ was measured using an enzyme-linked immunosorbent assay. (A) Functional sensitivity ( $p$ EC $\left.{ }_{50}\right)$ for each peptide ligand in the context of each $\mathrm{MHCl}$. Four replicate experiments are shown. Values below the limit of estimation were set to zero for graphical purposes and treated as missing data for statistical purposes. $P=0.0042$ for the ligand effect and $P=0.00069$ for the MHCl effect (two-way ANOVA with Tukey's post hoc test). (B) The agonist potency of FAT relative to the agonist potency of ELA expressed as $p E C_{50}{ }^{F A T}-p E C_{50}$ ELA, which is equivalent to the logarithm of the fold difference in functional sensitivity. Four replicate experiments are shown. Horizontal bars indicate median values. ${ }^{*} P<0.05$ (one-way ANOVA with Tukey's post hoc test). ( $C-E)$ Representative peptide titration experiment used to calculate the parameters in $A$ and $B$. Curves were fitted in Mathematica. All four replicate experiments are shown in SI Appendix, Figs. S4 and S5.

pCMV-VSV-G using Turbofect Transfection Reagent (Thermo Fisher Scientific). Lentiviral particles were concentrated using Lenti-X Concentrator (Takara Bio).

Generation of MEL5 TCR ${ }^{+}$CD8 $^{+}$J.RT3-T3.5 Cells. TCR-deficient J.RT3-T3.5 cells were transduced with MEL5 TCR lentiviral particles and magnetically enriched using anti-murine TCR- $\beta$-PE (clone REA318) in conjunction with anti-PE MicroBeads (Miltenyi Biotec). MEL5 TCR J.RT3-T3.5 cells were then transduced with CD8- $\alpha \beta$ lentiviral particles, and MEL5 TCR ${ }^{+}$CD8 ${ }^{+}$J.RT3-T3.5 cells were flow-purified using an Influx Cell Sorter (BD Biosciences).

Quantification of Activation-Induced CD69. C1R cells expressing comparable levels of HLA-A2 D227K/T228A, wild-type HLA-A2, or HLA-A2 A245V/K ${ }^{\mathrm{b}}$ were pulsed for $1 \mathrm{~h}$ with various concentrations of the indicated peptides. Cells were then washed twice with RPMI 1640 medium containing $100 \mathrm{U} / \mathrm{mL}$ penicillin and $100 \mu \mathrm{g} / \mathrm{mL}$ streptomycin and resuspended in R10. Each assay included $1.5 \times 10^{5}$ peptide-pulsed C1R cells and $5 \times 10^{4} \mathrm{MEL}^{2} \mathrm{TCR}^{+} \mathrm{CD} 8^{+}$ J.RT3-T3.5 cells. Unpulsed targets were used as negative controls. Expression of CD69 on the surface of MEL5 TCR ${ }^{+} \mathrm{CD}^{+}{ }^{+}$J.RT3-T3.5 cells was measured after $6 \mathrm{~h}$ using the following directly conjugated monoclonal antibodies: anti-CD8- $\alpha-P E-C y 7$ (clone RPA-T8; Thermo Fisher Scientific), anti-CD8- $\beta-$ eFluor660 (clone SIDI8BEE; Thermo Fisher Scientific), anti-CD69-BV421 (clone FN50; BioLegend), and anti-HLA-A2-FITC (clone BB7.2; BioLegend). Nonviable cells were excluded from the analysis using LIVE/DEAD Fixable Aqua (Thermo Fisher Scientific). Data were acquired using a NovoCyte Flow Cytometer (ACEA Biosciences) and analyzed using FlowJo software version 10.6.1 (FlowJo LLC).

Quantification of Activation-Induced IFN- $\gamma$ and MIP-1 $\beta$. C1R cells expressing comparable levels of HLA-A2 D227K/T228A, wild-type HLA-A2, or HLA-A2 $K^{b}$ were pulsed for $1 \mathrm{~h}$ with various concentrations of the indicated peptides. Cells were then washed twice with RPMI 1640 medium containing $100 \mathrm{U} / \mathrm{mL}$ penicillin and $100 \mu \mathrm{g} / \mathrm{mL}$ streptomycin and resuspended in R10. Each assay included $6 \times 10^{4}$ peptide-pulsed C1R cells and $3 \times 10^{4}$ clonal MEL5 or ILA1 $\mathrm{CD}^{+} \mathrm{T}$ cells. Unpulsed targets were used as negative controls. Supernatants were harvested after $4 \mathrm{~h}$ and evaluated for IFN- $\gamma$ or MIP-1 $\beta$ using enzymelinked immunosorbent assays (R\&D Systems).
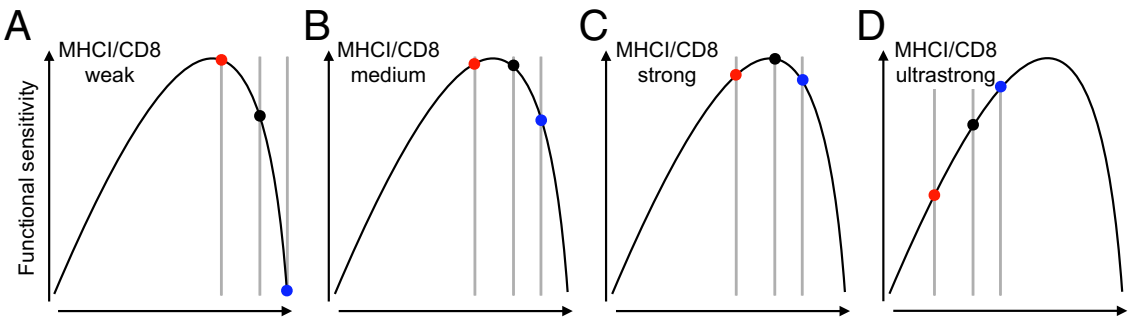

$\mathrm{TCR} / \mathrm{pMHCl}$ dissociation rate (logarithmic scale)

- ELIGIGILTV •ELAGIGILTV • FATGIGIITV

Fig. 3. Theoretical interpretation of the differential focusing effect mediated by CD8. Graphical representation of the differential focusing effect based on two key assumptions: 1) functional sensitivity depends nonmonotonically on the TCR/pMHCl dissociation rate; and 2) the pMHCI/CD8 interaction affects the $\mathrm{TCR} / \mathrm{pMHCl}$ dissociation rate by an invariant factor, equivalent to translation on a logarithmic scale (SI Appendix). ( $A-C$ ) Modulation of the $\mathrm{pMHCl} / \mathrm{CD} 8$ interaction moves peptide ligands along this curve, altering the agonist hierarchy as a function of the TCR/pMHCl dissociation rate. (D) A hypothetical ultrastrong $\mathrm{pMHCI/CD} 8$ interaction would be expected to reverse the agonist hierarchy from FAT $>$ ELA $>3 T$ to FAT $<$ ELA $<3$ T. 
Statistics. Functional assay data were processed using simultaneous nonlinear least squares parameter estimation encoded in Mathematica (23). Functional sensitivity $\left(p E C_{50}\right)$ was expressed as the decimal cologarithm $p$ of the $50 \%$ efficacy concentration $\left(\mathrm{EC}_{50}\right)$. Assay-derived estimates of $p \mathrm{EC}_{50}$ were treated as input data for subsequent analyses of variance. Data were analyzed using a one-way or two-way ANOVA with Tukey's post hoc test in Mathematica or Prism software version 8 (GraphPad).

Data Availability. All study data are included in the article and/or SI Appendix.

1. M. G. Rudolph, R. L. Stanfield, I. A. Wilson, How TCRs bind MHCs, peptides, and coreceptors. Annu. Rev. Immunol. 24, 419-466 (2006).

2. D. K. Cole et al., The molecular determinants of CD8 co-receptor function. Immunology 137, 139-148 (2012).

3. G. F. Gao et al., Crystal structure of the complex between human CD8 $\alpha \alpha$ and HLA-A2. Nature 387, 630-634 (1997).

4. R. Zamoyska, CD4 and CD8: Modulators of T-cell receptor recognition of antigen and of immune responses? Curr. Opin. Immunol. 10, 82-87 (1998).

5. G. F. Gao, B. K. Jakobsen, Molecular interactions of coreceptor CD8 and MHC class I: The molecular basis for functional coordination with the T-cell receptor. Immunol. Today 21, 630-636 (2000).

6. P. D. Holler, D. M. Kranz, Quantitative analysis of the contribution of TCR/pepMHC affinity and CD8 to T cell activation. Immunity 18, 255-264 (2003).

7. G. F. Gao et al., Classical and nonclassical class I major histocompatibility complex molecules exhibit subtle conformational differences that affect binding to CD8 $\alpha \alpha$. J. Biol. Chem. 275, 15232-15238 (2000).

8. J. R. Wyer et al., T cell receptor and coreceptor CD8 $\alpha \alpha$ bind peptide-MHC independently and with distinct kinetics. Immunity 10, 219-225 (1999).

9. H. A. van den Berg, L. Wooldridge, B. Laugel, A. K. Sewell, Coreceptor CD8-driven modulation of T cell antigen receptor specificity. J. Theor. Biol. 249, 395-408 (2007).

10. H. A. van den Berg et al., Cellular-level versus receptor-level response threshold hierarchies in T-cell activation. Front. Immunol. 4, 250 (2013).

11. B. Szomolay, T. Williams, L. Wooldridge, H. A. van den Berg, Co-receptor CD8mediated modulation of T-cell receptor functional sensitivity and epitope recognition degeneracy. Front. Immunol. 4, 329 (2013).

12. L. Wooldridge et al., Interaction between the CD8 coreceptor and major histocompatibility complex class I stabilizes T cell receptor-antigen complexes at the cell surface. J. Biol. Chem. 280, 27491-27501 (2005).

13. A. M. Kalergis et al., Efficient $T$ cell activation requires an optimal dwell-time of interaction between the TCR and the pMHC complex. Nat. Immunol. 2, 229-234 (2001)

14. A. Lanzavecchia, F. Sallusto, Dynamics of T lymphocyte responses: Intermediates, effectors, and memory cells. Science 290, 92-97 (2000).

15. H. A. van den Berg, N. J. Burroughs, D. A. Rand, Quantifying the strength of ligand antagonism in TCR triggering. Bull. Math. Biol. 64, 781-808 (2002).

16. M. Clement et al., Targeted suppression of autoreactive $\mathrm{CD} 8^{+}$T-cell activation using blocking anti-CD8 antibodies. Sci. Rep. 6, 35332 (2016).

17. D. Mason, A very high level of crossreactivity is an essential feature of the T-cell receptor. Immunol. Today 19, 395-404 (1998).

18. J. Ekeruche-Makinde et al., T-cell receptor-optimized peptide skewing of the T-cell repertoire can enhance antigen targeting. J. Biol. Chem. 287, 37269-37281 (2012).

19. M. Clement et al., Anti-CD8 antibodies can trigger $\mathrm{CD}^{+} \mathrm{T}$ cell effector function in the absence of TCR engagement and improve peptide-MHCl tetramer staining. J. Immunol. 187, 654-663 (2011).

20. M. A. Purbhoo et al., The human CD8 coreceptor effects cytotoxic $T$ cell activation and antigen sensitivity primarily by mediating complete phosphorylation of the $T$ cell receptor $\zeta$ chain. J. Biol. Chem. 276, 32786-32792 (2001).
ACKNOWLEDGMENTS. We thank Andrew Herman and Lorena Sueiro Ballesteros for assistance with cell sorting at the University of Bristol Flow Cytometry Facility. This work was funded by the Wellcome Trust (Grants WT079848MA and WT099067AIA). Additional support was received from the Horizon 2020 Research and Innovation Programme of the European Union via Marie Sklodowska-Curie Grant Agreement 721358 and from the Biotechnology and Biological Sciences Research Council (Grant BB/H001085/ 1). A.K.S. and D.A.P. were supported by Wellcome Trust Senior Investigator Awards.

21. T. Dockree et al., CD8 ${ }^{+}$T-cell specificity is compromised at a defined MHCI/CD8 affinity threshold. Immunol. Cell Biol. 95, 68-76 (2017).

22. L. Wooldridge et al., MHC class I molecules with superenhanced CD8 binding properties bypass the requirement for cognate TCR recognition and nonspecifically activate CTLs. J. Immunol. 184, 3357-3366 (2010).

23. L. Wooldridge et al., A single autoimmune T cell receptor recognizes more than a million different peptides. J. Biol. Chem. 287, 1168-1177 (2012).

24. D. K. Cole et al., Structural mechanism underpinning cross-reactivity of a CD8 ${ }^{+}$T-cell clone that recognizes a peptide derived from human telomerase reverse transcriptase. J. Biol. Chem. 292, 802-813 (2017).

25. A. K. Sewell, Why must T cells be cross-reactive? Nat. Rev. Immunol. 12, 669-677 (2012).

26. D. K. Cole et al., Hotspot autoimmune T cell receptor binding underlies pathogen and insulin peptide cross-reactivity. J. Clin. Invest. 126, 2191-2204 (2016).

27. R. Bosselut, T. I. Guinter, S. O. Sharrow, A. Singer, Unraveling a revealing paradox: Why major histocompatibility complex I-signaled thymocytes "paradoxically" appear as $\mathrm{CD} 4^{+} 8^{10}$ transitional cells during positive selection of $\mathrm{CD} 8^{+} \mathrm{T}$ cells. J. Exp. Med. 197, 1709-1719 (2003).

28. R. Maile et al., Peripheral "CD8 tuning" dynamically modulates the size and responsiveness of an antigen-specific T cell pool in vivo. J. Immunol. 174, 619-627 (2005).

29. D. Gangadharan, H. Cheroutre, The CD8 isoform CD8 $\alpha \alpha$ is not a functional homologue of the TCR co-receptor CD8 $\alpha \beta$. Curr. Opin. Immunol. 16, 264-270 (2004).

30. M. A. Daniels et al., CD8 binding to MHC class I molecules is influenced by T cell maturation and glycosylation. Immunity 15, 1051-1061 (2001).

31. A. M. Moody et al., Developmentally regulated glycosylation of the CD8 $\alpha \beta$ coreceptor stalk modulates ligand binding. Cell 107, 501-512 (2001).

32. M. A. Daniels, K. A. Hogquist, S. C. Jameson, Sweet ' $n$ ' sour: The impact of differential glycosylation on T cell responses. Nat. Immunol. 3, 903-910 (2002).

33. J.-H. Park et al., 'Coreceptor tuning': Cytokine signals transcriptionally tailor CD8 coreceptor expression to the self-specificity of the TCR. Nat. Immunol. 8, 1049-1059 (2007).

34. J. Huang et al., The kinetics of two-dimensional TCR and pMHC interactions determine T-cell responsiveness. Nature 464, 932-936 (2010).

35. P. P. Yachi, J. Ampudia, T. Zal, N. R. J. Gascoigne, Altered peptide ligands induce delayed CD8-T cell receptor interaction-A role for CD8 in distinguishing antigen quality. Immunity 25, 203-211 (2006).

36. D. K. Cole et al., Germ line-governed recognition of a cancer epitope by an immunodominant human T-cell receptor. J. Biol. Chem. 284, 27281-27289 (2009).

37. B. Laugel, D. A. Price, A. Milicic, A. K. Sewell, CD8 exerts differential effects on the deployment of cytotoxic T lymphocyte effector functions. Eur. J. Immunol. 37, 905-913 (2007)

38. L. Wooldridge et al., Enhanced immunogenicity of CTL antigens through mutation of the CD8 binding MHC class I invariant region. Eur. J. Immunol. 37, 1323-1333 (2007).

39. D. Sommermeyer, W. Uckert, Minimal amino acid exchange in human TCR constant regions fosters improved function of TCR gene-modified T cells. J. Immunol. 184, 6223-6231 (2010). 